\title{
Multi-Campus Universities Private-Cloud MIGRATION INFRASTRUCTURE
}

\author{
Abdel Rahman Alzoubaidi \\ Department of Computer Engineering, Al Balqa Applied University, Salt, Jordan
}

\begin{abstract}
Cloud Computing is an attractive research area for the last few years; and there have been a tremendous grows in the number of educational institutions all over the world who have either adopted or are considering migrating to cloud computing. However, there are many concerns and reservations about adopting conventional or public cloud based solutions. A new paradigm of cloud based solution has been proposed, namely, the private cloud based solutions, which becomes an attractive choice to educational Institutions. This paper presents the adjustment and implementation of private-based cloud solution for multi-campus educational institution, namely, Al-Balqa Applied University (BAU) in Jordan.
\end{abstract}

\section{KEYWORDS}

Cloud computing; Cloud computing provider; public-cloud; private cloud; cloud migration; multi-campus organization

\section{INTRODUCTION}

Cloud Computing is defined as the delivery of computing resources (hardware and software) as a service rather than a product, whereby resources are provided to users as a metered service over the Internet $[1,2]$. Cloud computing initially provides three main types of services; these are [36]:

- Infrastructure as a Service (IaaS): All required hardware to run a business is provided by CSPs and customers manage their application software.

- Platform as a Service (PaaS): A user pays to the service provider to use their platform as their IT solution. For example, if a user needs E-mail system or database software for their business, they can use a third party's computing service that prove email and database solutions.

- Software as a Service (SaaS): If a user needs to use a specific kind of software to get an output or to perform an analysis, then it is much cheaper to use that software service from a CSP rather than buying, installing and maintaining it.

Recently, new types of Cloud Computing services are emerged, namely, Data as a Service (DaaS) and Information Technology as a Service (ITaaS). DaaS is a cousin of SaaS. And it is based on the concept that the product, data in this case, can be provided on-demand to the user regardless of geographic or organizational separation of data provider and user [6]. ITaaS is a technologydelivery method that treats IT as a commodity, providing an enterprise with exactly the amount of hardware, software, and support that it needs for an agreed-on fee. In this context, IT encompasses all of the technologies for creating, storing, exchanging, and using business data. 
All of the above services can be accessed through one of two types of basic cloud-based solutions, namely, public and private cloud solutions [7, 8]. Public cloud is defined above and it has a number of advantages, including lower capital expenditures, employees work anywhere and focus on business not technology, no upgrades concerns, instant implementations, lower upfront and operation and maintenance costs, pay only for the required services, guaranteed service-level agreements (SLAs), predictable spending, and provide guaranteed scalability. However, in this implementation, computing services are usually provided as a service by a third-party, known as Cloud Service Provider (CSP). Therefore, security and privacy requirements are the main concerns in this type of implementation. Private cloud is similar to public cloud as it delivers the same services and has the same advantages except it offers its services through a proprietary infrastructure, which provides more security and privacy as compared to public cloud [9].

It has been well-recognized that educational institutions, among other organizations, can significantly benefit from Cloud Computing technological development and relax the many financial, operational, maintenance, management, and administrative challenges that are facing educational institutions, and meet users (e.g., students, faculties, staff, etc.) needs and maintain high satisfactory Quality-of-Service (QoS) $[4,5]$. In this research, we investigate and demonstrate how educational institutions, especially multi-campuses institutions such as Al-Balqa Applied University (BAU) in Jordan, can take advantage of the evolving Cloud Computing paradigms in providing high quality cost-effective IT services.

BAU is a multi-campus university having 13 distinct locations in Jordan. It serves around 45,000 students. BAU main campus in Salt is the center for all educational, administrative functions, services, and responsibilities related to all of its campuses. Currently, BAU employs a distributed computing IT infrastructure setup as it shall be discussed below, having its main Data Center (DC) at BAU main campus in Salt, and 12 regional DCs in remote campuses equipped with physical servers, storage and network resources.

Campuses are interconnected via either high speed fiber optic links or dedicated leased lines with different speeds, the connectivity is provisioned and managed by the Jordanian University Network (JUNet) and one of the main telecommunication service provider in Jordan (namely, Orange). In the current architecture, the IT resources are duplicated to ensure similar services across the main and remote campuses. This implies allocating a local administration and technical personnel for the main and each remote campus. Furthermore, the administration and technical team at the main campus should be qualified enough to provide technical, operational, administrative, and oversight across the university's main and remote campuses DCs.

The difficulty in deploying and updating core IT services across the main and remote campuses, legacy and inefficiency of existing design, and cost of resource duplication (hardware, software, and human resources) to operate, maintain, and secure the existing campus-based IT services. Moreover, the existing design is unable to provide the agility, elasticity, rapid deployment, and QoS necessary to cope with the changing IT requirements for the students, the faculties, and the staff. In fact, any improper management may end up with significant expenditure in purchasing and maintaining unplanned IT resources for each campus. BAU Computer Center Directors frequently find they need to acquire IT resources for remote campuses to fulfill unplanned or short-term requirements, such as at some peak time at the start and at the end of each semester.

This paper presents the roadmap to alleviate the current DC redundancies and overheads in BAU campuses and ensure high reliability, availability, and business continuity through moving to private cloud implementation, which is referred to as Balqa Private Cloud (BPC). BPC consolidates BAU IT services and infrastructures into one corporate fold to allow for management and delivery of all common IT resources and services. 
Moreover, the new BPC setup mitigates the problem of single points of failures at each DC level concerning its physical servers, WAN connectivity with the main DC; local and remote backups from remote campuses to the central DC that is continuously increasing computing and storage resources. BPC preserves control of services delivery such as centralized Internet and security and privacy services, policies, application maturity, performance requirements, and business differentiation reasons.

This paper is divided into five main sections. This section introduces the main theme of the paper. A dedicated literature review is given in Section 2. Section 3 presents a detailed description of the existing infrastructure at BAU. Section 4 is devoted for the description of the migration phases from the existing distributed DCs to private cloud infrastructure. Finally, in Section 5, conclusions recommendations for future work are pointed-out.

\section{LITERATURE REVIEW}

\subsection{Cloud Computing Definition}

Cloud Computing is a model for enabling suitable, on-demand network access to a shared pool of configurable computing resources (e.g., servers, storage, applications, and services) that can be quickly provisioned and released with minimal management effort or service provider interaction. It is also defined as a model of hardware and software deployment where the software application is hosted on extremely high performance hardware, and provided as a service to customers across the Internet [1]. By eliminating the need to install and run the application on the customer's own servers or premises, the burden of software maintenance, ongoing operation and support has been removed. Required IT resources are paid for through a subscription instead of a license; where businesses no longer require to procuring and installing costly IT resources on their premises [2]. As we have discussed above that Cloud Computing provides three main services with more services were lately emerged. Fig. 1 shows the current Cloud Computing services that are currently provided by most major hardware and software brands (e.g., HP, DELL, Amazon, IBM, Google, and Microsoft, etc.) [10].

Fig. 1. Cloud Computing services.

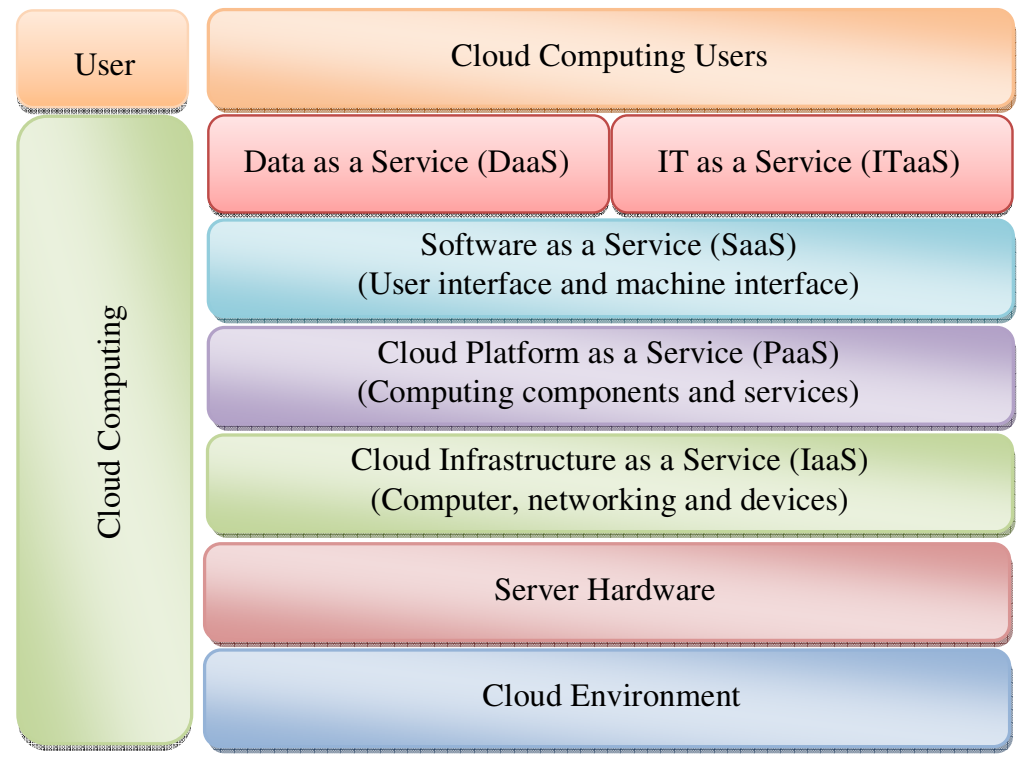


International Journal on Cloud Computing: Services and Architecture (IJCCSA) Vol. 6, No. 3, June 2016

\subsection{Forms of Cloud Computing}

Cloud Computing comes in three forms: public-, private-, and hybrid-cloud, in what follows, we shall provide a brief introduction for each of them.

\section{A. Public cloud}

Public cloud is one in which the services and infrastructure are purchased by an organization and delivered via the Internet by a third-party provider, usually CSP. These services use storage capacity and processor power that is not owned by the organization itself. Instead, this capacity owned either by the primary vendor or by a cloud infrastructure vendor. These clouds offer the greatest level of efficiency in shared resources; however, they are also more vulnerable than private cloud $[7,8]$.

\section{B. Private cloud}

Private cloud is one in which the services and infrastructure are maintained on a private network; therefore, it is also known as an internal- or enterprise-cloud, as it resides on the organization's Intranet or DC where all data is protected behind a firewall. These clouds offer the greatest level of privacy, security, and control, but they require the organization to still purchase and maintain all the software and infrastructure, which slightly increases the system initial cost. Private cloud is in general more promising than the public cloud due to the concerns of control, vendor lock, security, privacy, and reliability [11].

\section{Hybrid cloud}

A hybrid cloud is an integrated cloud service utilizing both private and public clouds to perform distinct functions within the same organization. By spreading things out over a hybrid cloud, each aspect at the organization is kept in the most efficient environment possible. The downside is that organization has to keep track of multiple different security platforms and ensure that all aspects of the organization can communicate with each other [12].

There is a tendency in many organizations and educational institutions to invest in different cloud models, such as private, public, or hybrid services [13].

\subsection{Application of Cloud Computing in Educational Institutions}

One of the most important applications of Cloud Computing is in education. In the new globalized economy, educational institutions and universities must provide high quality IT learning infrastructure and prepare students for the growing challenges of the $21^{\text {st }}$ Century. This is especially true in multi-campus universities that have various campuses distributed across wide areas, where such IT infrastructure must be provided for each campus [14].

It has been recognized that Cloud Computing is well suited for multi-campus educational institutions; as it provides high availability, scalability, higher performance, up-to date hardware, instant software updates, and a wide access to different academic resources, applications, and educational tools. It also provides cost-effective solution to the learning process, more agility, foster development by improving collaboration between students and educators, more efficient administration and less expenditure on operation, maintenance, and management [15]. Furthermore, Cloud Computing opens a new era and improves the quality and effectiveness of social applications in universities, such as a cloud-based storage of social academic networks between members of higher education: professors, students and researchers. They can share common conference interests, peer review relationships, joint publications, and locate academic library resources effectively [16]. 
Cloud Computing can play a big role in developing one of the most promising paradigms for education, namely, e-learning [17]. E-learning is depending on the access to the Information and Communications Technology (ICT). But the infrastructure support for the e-learning system and its maintenance require high costs [18]. Cloud Computing is a better solution, providing low costs, higher performance, instant software updates and a wide access to different academic resources, applications and educational tools. To complete tasks and assessments, the users can run the applications from cloud through their PC, mobile phones, tablet PC, smart phones. Cloud Computing can help search within the growing resources, find and manage the information easier and faster, and provides access to information from other locations [19].

Cloud Computing is applied to digital library projects to improve the library management system for upgrading, rebuilding or integrating with other systems [4]. It also applied to e-science applications as they become larger and more complex, requiring access to vast amounts of computational resources, specialized software, remote instruments and access to distributed data. Thus, moving them to Cloud Computing infrastructures can lead to faster execution. The escience applications of the future may therefore be implemented for execution over cloud resources which are the predominating enabler for e-science applications of the future [20].

Shunye et al. [21] analyzed the development of the cloud computing in education, proposed a new e-learning architecture based on private cloud, and presented the expected benefits from the proposed architecture. They conclude that educational institutions can significantly benefit from Cloud Computing by increasing the benefits of students, teachers and administrators.

Getso and Ahmed [4] reviewed the services that can be provided by Cloud Computing infrastructure in the educational arena, especially in the universities where the use of computers are more intensive and what can be done to increase the benefits of common applications for students and teachers, and how to develop the system that can be work in any time of cloud like private cloud, public cloud, and hybrid cloud. Praveena and Betsy [22] provided a comprehensive introduction to the application of Cloud Computing in universities.

For more detailed information concerning the emergence and popularity, essential characteristics, services and deployment models, advantages and disadvantages of Cloud Computing and private cloud can be found in [23-25].

\section{BAU EXISTING IT INFRASTRUCTURE}

BAU existing IT infrastructure is designed for campus-based services as shown in Fig. 2, where each campus has its own setup. BAU was interconnected with JUNet and/or Orange networks for Internet and data services. The interconnection speeds between the main DC and regional campuses DCs are based on the providers' resources availability in a form of one connection. The link speed choices are limited to two factors; the resources availability and the required QoS required by the connected campus. BAU main campus and Faculty of Engineering and Technology (FET) are provided by 1 Gbps fiber optic connections. While, regional campuses interconnection links are ranging from 1, 4, 8, to $16 \mathrm{Mbps}$. No redundant links are provided, except a single $8 \mathrm{Mbps}$ connection to the main campus. 


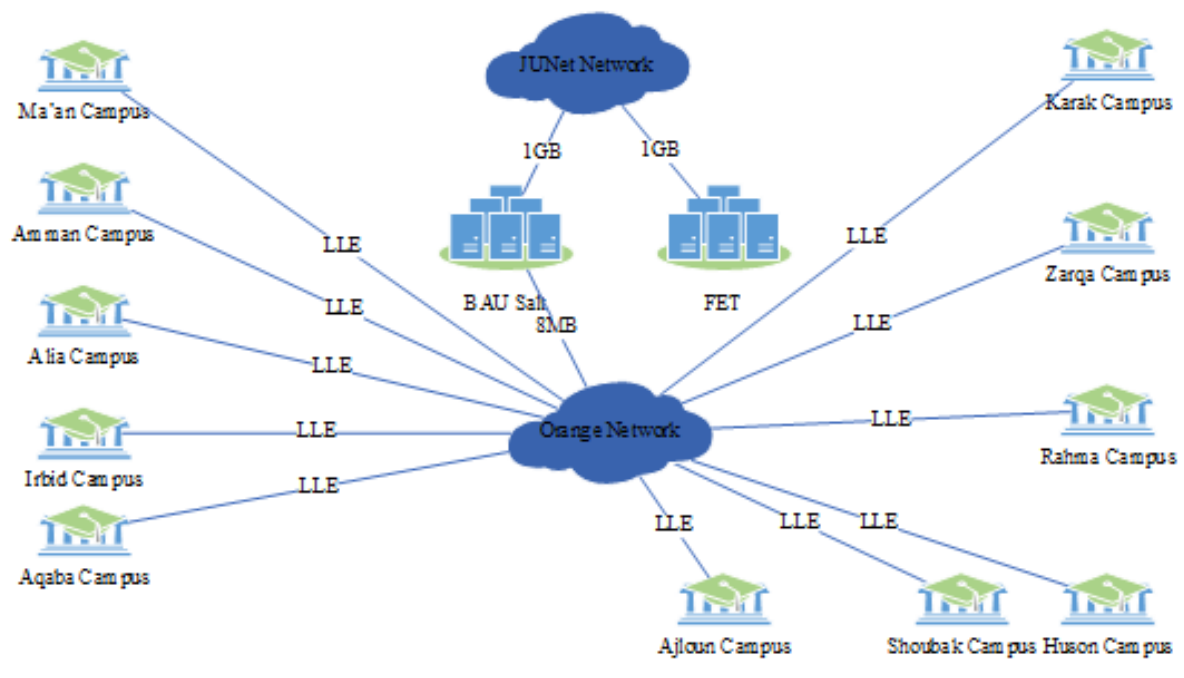

Fig. 2. BAU main campus DC with regional campuses DC connectivity.

\subsection{Limitations and Challenges}

BAU existing campuses infrastructure is extremely inflexible, old and inefficient to suite current IT services needs and requirements. Usually it cannot provide the agility, elasticity, rapid deployment and QoS necessary to run the changing IT requirements for the students, staff and administration members. The resource duplication in the regional campuses is also a significant disadvantage.

The number of student enrolled in the different campuses per semester is not precisely known. Thus it is a difficult task for the IT department in each campus to scale the IT system to meet the future demands. Accordingly, the infrastructure is over-provisioned by the IT administration, in order to guarantee an acceptable QoS and to remain competitive. For instance, a known problem in a DC is the scaling of the on-line registration Web application. After it is already up and running, new demand often arises and exceeds the initial predictions. Only then it becomes clear that the computing power of the servers is not enough and the infrastructure is not flexible enough to allow for quick adjustments leading to unforeseen cost.

When the IT department asked to deliver a high quality service to the student, they acquire the necessary computing resources and then build an infrastructure that is specific to the service requirements. This procedure can result in an increase in delivery time, higher costs because of duplicate resources, and unmet university expectation of agility and cost reduction.

In addition, the already busy staff in regional campuses has to cope without having a proper IT support. This ends up costing the employees a lot of time, energy and motivation which was intended to be focused on the service. The improper management ends up in expenditure in purchasing and maintaining unplanned IT equipment. Adopting public or private cloud based computing will put an end to this and similar scenarios. The IT computing services become a resource easily provisioned when needed and released afterwards. 
The Cloud Computing paradigm in IT can bring benefits regarding efficiency and flexibility to universities including multi-campus of any focus or size. Being a multi-campus university is a strong reason for BAU to migrate to Cloud Computing based infrastructure, the maintenance and upgrade of IT infrastructure for all its campuses is very expensive. Recruiting trained certified skilled staff for each campus. Software licenses for multi DCs equipped with physical servers and support multi-campus agreement and infrastructure computing resources needs huge yearly investment and continuous distributed efforts for upgrade and maintenance and management responsibilities across all campuses. Migration is projected to discharge the employees to fully concentrate on the core business activities for building and strengthening organizational strengths. Moreover, it also enables BAU computing resources to become more agile and adaptable for dealing with the university financial turbulences, and to provide the benefits of the private cloud as well as control their own destiny and guarantees for their internal security and SLA requirements.

BAU legacy DCs infrastructure can no longer meet the increasing demands of current modern DC services. Virtualization, Cloud Computing and the deployment of new applications that require agility and high-performance computing cannot perform efficiently on the existing old enterprise storage, servers, and networking DC infrastructure.

Based on the above discussion, there are several institutional and technology related reasons for BAU to move its current IT infrastructure from campus-based to Cloud Computing based infrastructure. For example, lower cost of entry, reduced risk of IT infrastructure failure, higher Return-of-Investment (RoI), quick responses to changes in demand, rapid deployment, increased security, and ability to focus on an organization' core business.

\section{BAU Migrating To Private Cloud}

In this research, we started with a systematic assessment of the existing BAU IT infrastructure, including computing resources, storage, internetworking facilities, and applications. The assessment process was looking for the consolidation of the existing DCs into a managed BPC. There are many issues that should be considered and made conscious decisions about, before deciding on the optimum solution to overcome the limitations and meet the challenges discussed in the previous section, e.g., available resources, the applications need to move into the cloud. It is necessary to work with the existing resources, partners, developers, and any other stakeholders to understand and asses their services and capabilities and how to align and integrate them all together to achieve optimum system design. Certainly, there is a big tendency to look at technology aspect to provide emulation and virtualization, service task interfaces to satisfy the on demand services, work with capacity planning, performance management and resources utilization.

As we discussed earlier that BAU campuses are inter connected via either high speed fiber links or dedicated leased lines with different speeds, the connectivity is provisioned and managed by JUNet and Orange. Furthermore, many network architectures include a tiered design with three or more tiers such as core, distribution, and access. Designs are driven by the port bandwidth and quantity required at the edge, as well as the ability of the distribution and core tiers to provide higher speed uplinks to aggregate traffic.

The basic model that we propose to overcome the limitations and meet the challenges we face in the existing BAU IT infrastructure is the provision of Private Cloud, which allows users to choose the best mix for their needs to achieve an effective, scalable, and on demand Private Cloud managed services with infrastructure and resources to help the university gain a competitive edge through greater agility, lower costs, and reduced risk. 
BPC is the heart of our solution to consolidate the DCs IT infrastructures into the corporate BPC to allow the use of common IT resources and management whereas reducing DC redundancies and services overhead. Since, the current local infrastructure is inefficient, obsolete, inflexible, and has a high total cost of ownership. The cloud technology service layers, such as Infrastructure as a Service (IaaS), Platform as a Service (PaaS), and eventually Database as a Service (DaaS) offer an excellent solution to overcome these problems [1-4].

The main issues that have been considered to ensure maximum possible QoS include: inter-cloud, workload mobility, system recovery, data replication, multi-tenancy, and redundant components and network paths [23-25].

\subsection{Private Cloud Interconnections}

Fig. 2 represents the physical servers, the physical Gbps backbone, network links and SAN storage. This comprises BAU DCs to be located at the BAU main campus and FET campus around $30 \mathrm{Km}$ a part from the main campus. The basic model for private cloud provision is applied to offer regional campuses the best mix for their needs with regard to private cloud managed services. BPC adopted a fully managed private cloud that includes the services above and below the hypervisor. Also, it will feature reusable service profiles that can store configurations, which can be adapted with ease to conform to new requirements and provide a pool of networkable system service that is on demand, accessible, measured pool of service that is provided in an elastic and scalable fashion on BAU premises.

\section{A. Migration Phase \#1}

In Phase \#1, BAU multi-campus interconnections are upgraded according to the providers' availability and the respective campus QoS requirements as mentioned above. Five new high speed links to regional campuses are provided with $1 \mathrm{Gbps}$ fiber optic links. Four of these fiber optic links are connected to the National Backbone Network (NBN) and then connected to the BAU main campus DC through 1 Gbps link, while the fifth fiber optic link is connected to JUNet and then through 1 Gbps link to BAU main campus DC as shown in Fig. 3. All other interconnection links are unchanged.

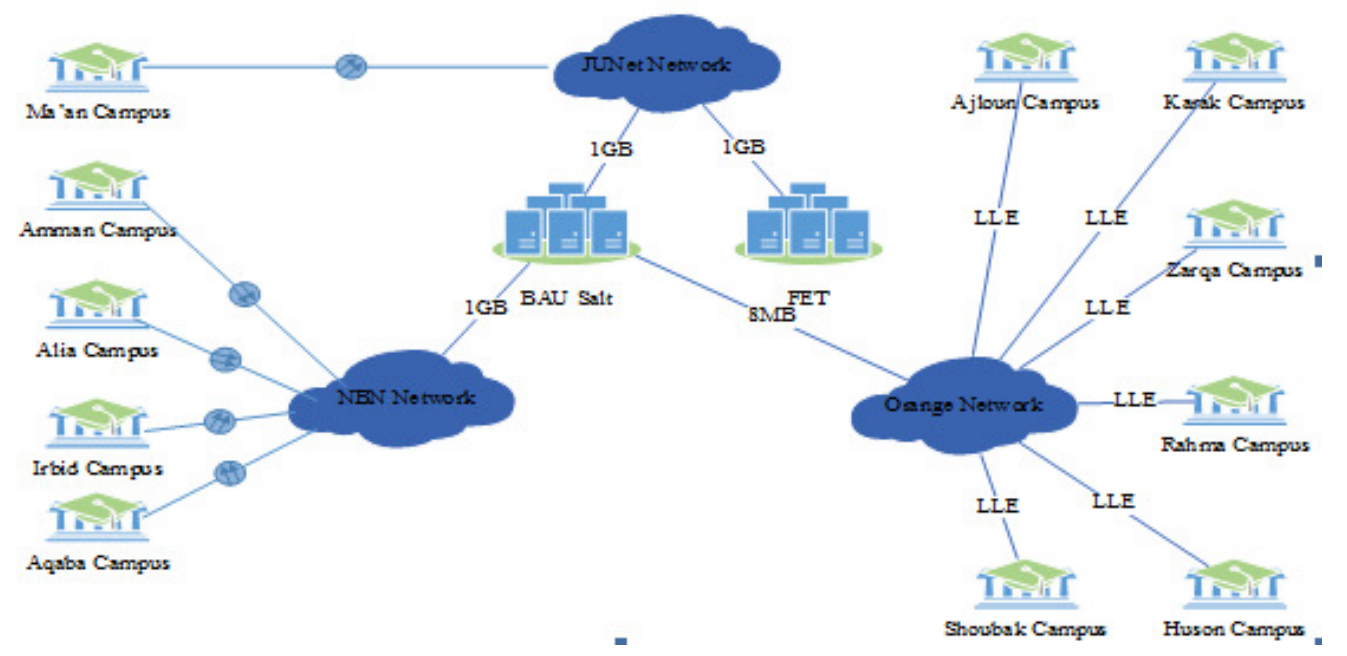

Fig. 3. Phase \#1 migration. 
It can be clearly seen from Fig. 3 that the main campus DC is accessed through different networks, JUNet, NBN Network, and Orange Network, which provides higher access bandwidth (better QoS) for the regional campuses, and eliminate the problem of single point of failure of the original design. For example, the $8 \mathrm{Mbps}$ data link between BAU main campus DC provided by orange is shared between 6 regional campuses instead of 11 regional campuses in the original design. However, still in this migration phase, the FET DC cannot access directly by any regional campus, except one, where the others can only access FET DC through BAU main campus DC.

\section{B. Migration Phase \#2}

In Phase \#2, more reliable high speed links are installed for interconnecting regional campuses, where five of the six remaining campuses from Phase \#1 are connected to Orange Network through high speed 1 Gbps fiber optic link. One campus, namely, Shoubak Campus, remains an upgraded due to unavailability of fiber connections. In Phase \#2, BAU multi-campus interconnections are improved with the provision of additional $100 \mathrm{Mbps}$ and upgrading the existing $8 \mathrm{Mbps}$ link to $100 \mathrm{Mbps}$. The new $100 \mathrm{Mbps}$ is used to directly interconnect FET DC to the regional campuses through the existing Orange Network infrastructure, as shown in Fig. 4.

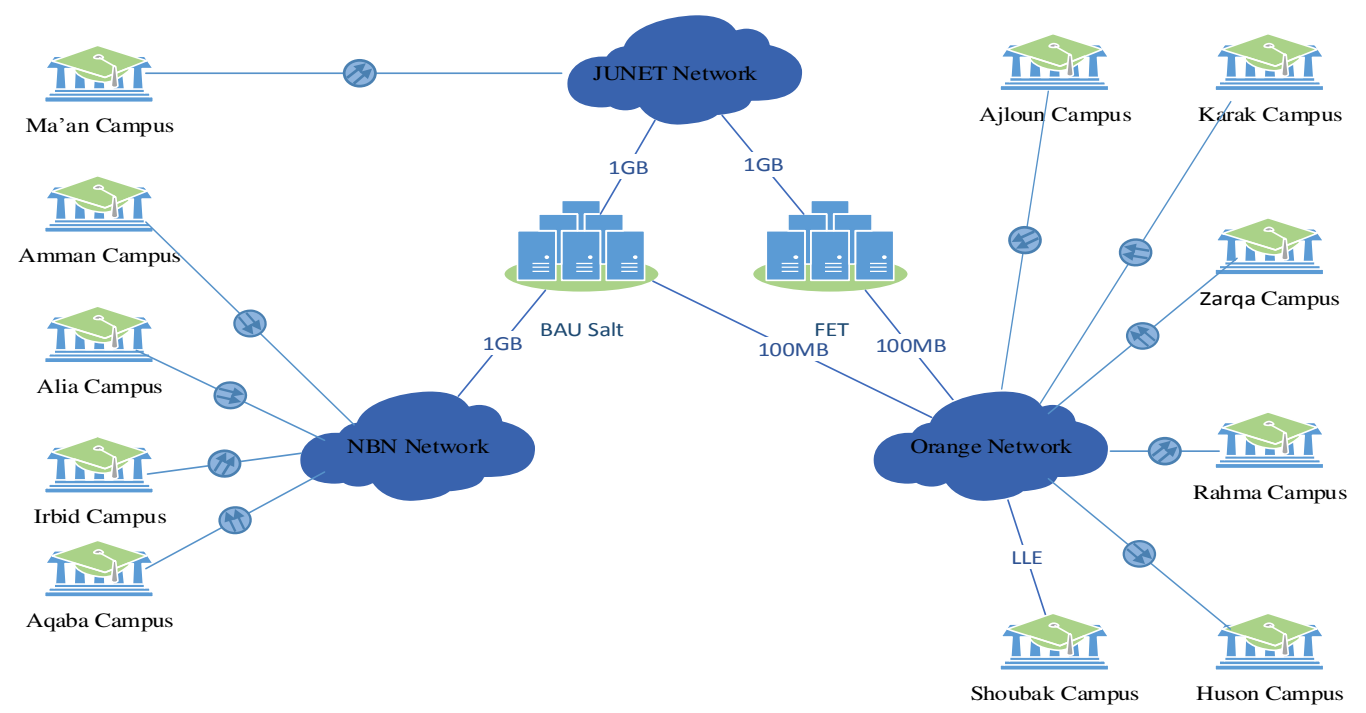

Fig. 4. Phase \#2 migration.

Upon completion of Phase \#2, it can be clearly noticed that all regional campuses are connected to the Main DC as well as to FET DC to ensure higher network reliability and continuous availability. Fig. 4 shows that the single point of failure for the connection links in Fig. 2 is eliminated, where we the regional campuses are divided into three groups connected to the main DC through three different providers, namely, Orange, NBN, and JUNet.

\section{Migration Phase \#3}

$\mathrm{BPC}$ requires reliable and available interconnection to provision its centralized services to all campuses. In this direction, in Phase \#3, we establish a backup connection link to all campuses. The primary campus interconnections are 1 Gbps speed links among regional campuses. To achieve the desired high availability services requirement a 4 Mbps backup redundant links are in place as presented in Fig. 5 to eliminate BPC inter-campus disconnectivity. 


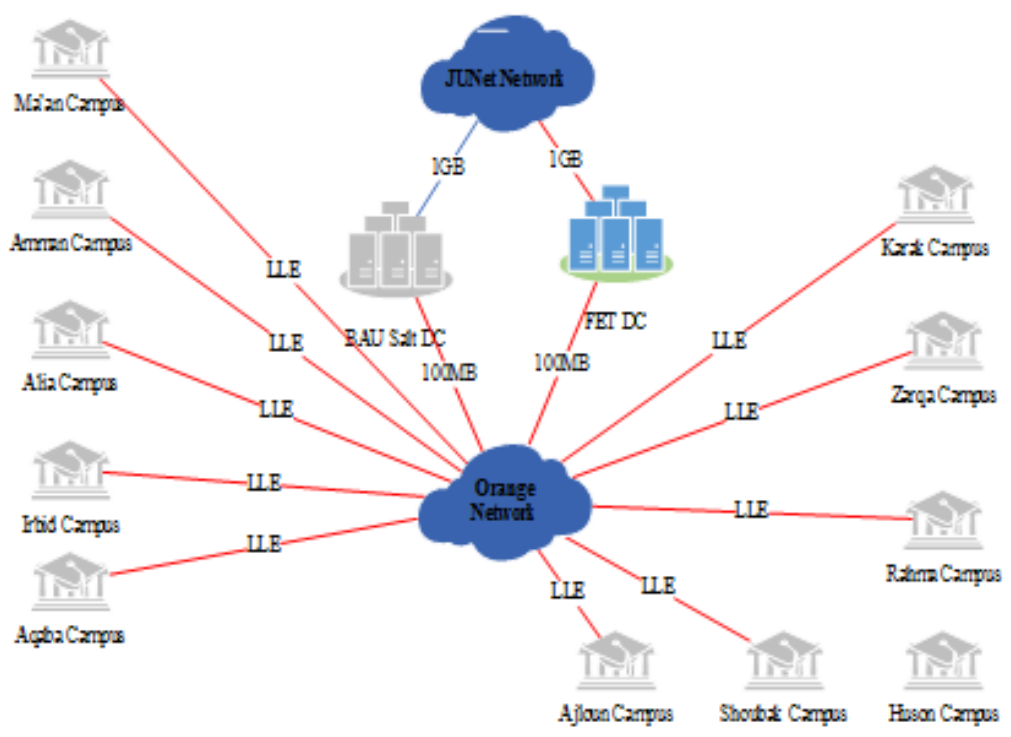

Fig. 5. Phase \#3 migration.

Upon completion of Phase \#3, all regional campuses DC are connected to the Main and FET DCs through two links, one is a dedicated 1 Gbps fiber link and the other is a 4 Mbps leased lines achieving higher reliability and availability.

\subsection{Private Cloud Data Center}

As it has been discussed above, BAU used to employ 13 DCs to host the databases and applications servers and internal or external storage units of the Main DC, FET DC, and 11 regional DCs. These resources are physical servers, either centralized to serve all campuses or centralized dedicated to each campus. Existing of a reliable interconnection network is one of the major requirements for building a reliable cloud service. Thus, upon completion of the interconnection network discussed above, we found ourselves that we are in a position to build our own private cloud infrastructure and consolidate our DCs into two VDCs, namely, the Main DC at main campus and FET DC $30 \mathrm{Km}$ apart with full confident, as shown in Fig. 6. The consolidated two DCs form the core of BPC migration infrastructure for provisioning of our cloud services and provide full backup facilities for disaster recovery (DR). 


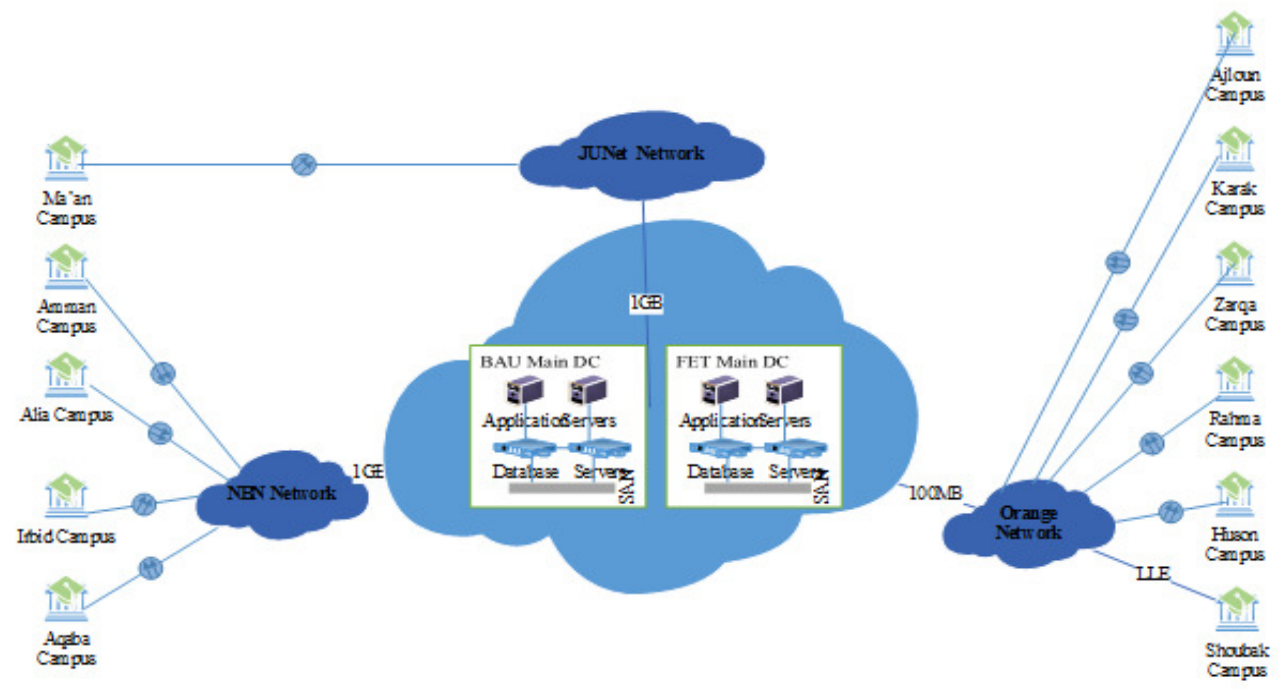

Fig. 6. BAU Private cloud (BPC).

\section{Conclusions}

This paper presents a migration approach, namely, phase-based migration approach, for a multicampus BAU from using distributed DCs at its main and regional campuses to private cloud based solution and building its own BPC to provision ITaaS. Moving to private cloud reduces the universities' IT administration, maintenance, and software licensing costs, at the same time, private, personal and sensitive information of universities are protected.

The phase-based migration approach adopted in building BPC proves to be the best approach to follow due to limitation in resources as well as to not to interrupt the University services, where lots of students and staff need to access IT resources continuously. Furthermore, it provides us with distinct milestones to test and evaluate our assets migration process to utilize cloud infrastructure.

The development and consolidation of resources for BAU into a successful private cloud opens away for providing ITaaS for all regional campuses without any regional DC resources. Furthermore, uses the above experience and views as to how to migrate to implement a shared configurable private infrastructure services across a multi-campus university or a consortium of universities.

\section{REFERENCES}

[1] Erl, T.; Puttini, R.; Mahmood, Z. (2013). Cloud Computing: Concepts, Technology \& Architecture. Publisher: Thomas Erl, ${ }^{\text {st }}$ Edition.

[2] Cervone, H. F. (2010). An overview of virtual and cloud computing. OCLC Systems \& Services, 26(3), 162-165.

[3] Kavis, M. J. (2014). Architecting the Cloud: Design Decisions for Cloud Computing Service Models (SaaS, PaaS, and IaaS). John Wiley, $1^{\text {st }}$ Edition.

[4] Bucsa, R. C., \& Ocneanu, L. (2014). Cloud computing technology in educational institutions. Economy Transdisciplinarity Cognition, 17(1), 98-102.

[5] Getso, M. and Ahmed, R. (2014). Applications of cloud computing in academic institutions. International Journal of Information Systems and Engineering, 2(1), 65-72. 
International Journal on Cloud Computing: Services and Architecture (IJCCSA) Vol. 6, No. 3, June 2016

[6] Rajesh, S.; Swapna, S.; \& Reddy, P. S. (2012). Data as a Service (Daas) in Cloud Computing [DataAs-A-Service in the Age of Data]. Global Journal of Computer Science and Technology, 12(11), 2529.

[7] Pantic, Z.; \& Babar, M. A. (2012), Guidelines for building a private cloud infrastructure. Technical Report TR-2012-153, IT University of Copenhagen, Denmark.

[8] Doelitzscher, F.; Sulistio, A.; Reich, C.; Kuijs, H.; \& Wolf, D. (2011). Private cloud for collaboration and e-learning services: From IaaS to SaaS. Computing Archives for Informatics and Numerical Computation, 91(1), 23-42.

[9] Bender, D. (2012). Privacy and Security Issues in Cloud Computing. Computer and Internet Lawyer, 29(10), 1-15.

[10] Al-Bahadili, H., Al-Sabbah, A., and Abu Arqoub, M. (2013). The architecture and analysis of a new cloud collaborative commerce model. International Journal of Cloud Applications and Computing (IJCAC), 3(3), 1-19.

[11] Kumar, S., Srivastava, S., Kumar, V., Kumar, O., \& Singh, A. K. (2012). Private Cloud: A paradigm of cloud computing with university shared data center (USDC). International Journal of Computer \& Communication Technology (IJCCT), 3(6, 7, 8), 117-121.

[12] Zhang, H. Jiang, G., Yoshihira, K.,Chen, H. (2010). Intelligent workload factoring for a hybrid cloud computing model. The World Conference on Services, Los Angeles, CA, 6-10 July 2010, 701-708.

[13] Wei-Wen Wu, W-W., Lan, L.W., \& Lee, Y-T. (2013). Factors hindering acceptance of using cloud services in university: a case study. The Electronic Library, 31(1), $84-98$.

[14] Thomas, P.Y. (2011). Cloud computing: A potential paradigm for practicing the scholarship of teaching and learning. The Electronic Library, 29(2), 214-224.

[15] Mircea, M., \& Andreescu, A. I. (2011). Using cloud computing in higher education: A strategy to improve agility in the current financial crisis. Communications of the IBIMA, Vol. 2011, Article ID 875547.

[16] Tam, W., Cox, A.M., \& Bussey, A. (2009). Student user preferences for features of next-generation. OPACs: A case study of University of Sheffield International Students. Program: electronic library and information systems, 43(4), 349-374.

[17] Kirkwood, K. (2010). The SNAP Platform: Social networking for academic purposes. Campus-Wide Information Systems, 27(3), 118-126.

[18] Uptal, J. B. \& Majidul A. (2013). E-Learning using Cloud Computing. International Journal of Science and Modern Engineering (IJISME), 1(2), 9-13.

[19] Divya, P. and Prakasam, S. (2015). Effectiveness of cloud based e-learning system (ECBELS). International Journal of Computer Applications, 119(6), 29-36.

[20] Mustafee, N. (2010). Exploiting grid computing, desktop grids and cloud computing for e-science: Future directions. Transforming Government: People, Process and Policy, 4(4), 288-298.

[21] Shunye, W., Dayong, L., and Zijuan, Z. (2014). E-Learning system architecture based on Private Cloud for university. Journal of Chemical and Pharmaceutical Research, 6(5), 492-498.

[22] Wang Shunye, Liu Dayong, and Zhang Zijuan. E-Learning System Architecture based on Private Cloud for University. Journal of Chemical and Pharmaceutical Research, Vo. 6, No. 5, pp. 492-498, 2014.

[23] K. Praveena and T. Betsy. Application of Cloud Computing in Academia. IUP Journal of Systems Management, Vol. 7, No. 3, pp. 50-54, 2009.

[24] Khmelevsky, Y. \& Voytenko, V. (2010). Cloud Computing Infrastructure Prototype for University Education and Research. 2010_WCCCE Proceedings of the $15^{\text {th }}$ Western Canadian Conference on Computing Education.

[25] Zhang, Q., Cheng, L., and Boutaba, R. (2010). Cloud computing: state-of-the-art and research challenges. Journal of Internet Services and Applications, 1(1), 7-18. 
International Journal on Cloud Computing: Services and Architecture (IJCCSA) Vol. 6, No. 3, June 2016

\section{AUTHOR}

Abdel Rahman Alzoubaidi is Associate Professor at Computer Systems Engineering Department and Computer Center Director at Al-Balqa Applied University. Alzoubaidi studied Automations ad Computer Engineering at the Technical University of Iasi and then left for Mu'tah University where he worked as teaching assistant on a scholarship where he obtained his MPhil and DPhil in data Communications and Computer Networks in 1996 under the supervision of Professor Ed. Powner. He subsequently joined the Department of Computer Engineering at Mu'tah University as faculty where he became

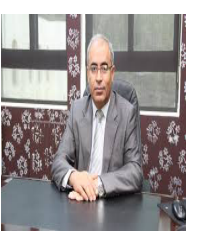
Associate Professor in 2004 and served as Director of Computer Center from 1996 to 2007. He was appointed President's Assistant for ICT. His research interests center on improving the understanding, design, and performance evaluation of networked computer systems, Cloud Computing, eLearning, Cyber Security, Wireless and Ad-hoc networks. He worked as Information and Communication Technology consultant for the Ministry of Education. He designed and implemented many innovative ICT projects in Jordan. He has given numerous invited talks and tutorials, and is a consultant to companies involved in Internet technologies. 